In severe forms of the disease, with considerable lateral displacement or subluxation of the femoral head, infraction of part or all of the uncovered area of the epiphysis, widening of the epiphysial nucleus, or a slow rate of healing, longcontinued conservative treatment is needed or, as has recently been suggested, surgical intervention by upper femoral ${ }^{67}$ or innominate osteotomy. ${ }^{8}$ It is still too early to assess the true results of such treatment. Techniques such as drilling of the growth plates, fenestration, and bone grafting have all been popular at one time and have all been rejected ultimately. Femoral or innominate osteotomy, which aims to improve the containment of the femoral head, appears to have a logical basis for its performance, but only the longterm follow-up of sufficient numbers of severely affected hips will show whether these procedures will fulfil the high hopes of their authors.

1 Catterall, A., fournal of Bone and foint Surgery, 1971, 53-B, 37.

Zahir, A., and Freeman, M. A. R., fournal of Bone and foint Surgery, 1972, 54-A, 125.

3 Kemp, H. B. S., Annals of the Royal College of Surgeons of England, 1973, 52,18 .

4e Valderrama, J. A. F., fournal of Bone and foint Surgery, 1963, 45-B, 462 .

5 Jacobs, B. W., Fournal of the American Medical Association, 1960, 172, 527

- Axer, A., fournal of Bone and foint Surgery, 1965, 47-B, 489.

Somerville, E. W. Fournal of Bone and foint Surgery, 1971, 53-B, 639.

8 Canale, S. T., D'Änca, A. F., Cotler, J. M., and Snedden, H. E., Fournal of Bone and foint Surgery, 1972, 54-A, 25.

\section{Renal Carbuncle}

Debilitation is a classical precursor of renal carbuncle, and so is diabetes. Most reported cases were in young adults before the days of penicillin. But in recent years we have grown used to the idea that the disease is extinct-a condition only to be seen at surgical examination and then in pots well bleached by age. There are signs that this happy interlude is over. In 1968 O. E. Cobb ${ }^{1}$ reported four cases of the condition to remind surgeons that it still could occur. Now it appears that debilitated drug addicts, who give themselves unclean intravenous injections through dirty needles, are specially prone to this disease. ${ }^{2}$

The organisms reach the kidney through the blood stream. Classically they are staphylococci derived from distant boils, septic fingers, chest infections, osteomyelitis, and infected abrasions and insect bites. Other organisms can cause an identical lesion. J. G. Rabinowitz and colleagues report two men who developed a carbuncle from coliform organisms probably originating in prostatitis. ${ }^{2}$ In several series ureteric obstruction seems to play a part in the origin of the carbuncle, though the pattern of inflammation in the kidney is different from usual. Unlike the common suppurative pyelonephritis, in which it spreads in a distinct wedge outwards from calices, in carbuncle the blood-borne organism forms a cortical abscess, and the infection spreads out into adjacent parenchyma to form a more or less confluent collection of abscesses without any well-defined wall. In contrast to pyelonephritis the suppurative lesion of the carbuncle is not in communication with the renal collecting system. When it spreads it does so centrifugally and forms a perinephric abscess.

Patients present with the general features of a severe infection with fever and rigors, a raised erythrocyte sedimentation rate, and a polymorphonuclear leucocytosis. They have no urinary symptoms as a rule, and the urine is free from pus or organisms, but pain in the loin may suggest trouble in the kidney. A plain radiograph shows the spine curved away from the side of the lesion, the psoas border is blurred, and the renal shadow is obscured by soft tissue swelling. The excretion urogram may show delayed or even absent excretion, but it may disclose a soft tissue mass displacing and compressing adjacent calices.

These features may suggest renal carcinoma, especially in a patient given antibiotics to overcome acute inflammation. An arteriogram may help to make the diagnosis, especially in the early stages, for no tumour circulation will be seen and the renal vessels will be shown displaced by the soft tissue lump. In more chronic cases, and later on in the course of the disease, angiography may cause confusion by showing inflammatory vessels that may be mistaken for those of a neoplasm. . $^{3-5}$

The best treatment is by antibiotics, though coexisting ureteric obstruction may need to be dealt with specifically. The antibiotics must be kept up until all the clinical and radiographic features have disappeared, when the kidney may be expected to return to normal.

1 Cobb, O. E., British fournal of Urology, 1966, 38, 262.

2 Rabinowitz, J. G., Kinkhabwala, M. N., Robinson, T., Spyropoulos, E., and Becker, J. A., American fournal of Roentgenology, 1972, 116, 740. ${ }^{3}$ Caplan, L. H., Siegelman, S. S., and Bosniak, M. A., Radiology, 1967, 88, 14.

4 Caro, G., Meisell, R., and Held, B., Radiology, 1969, 92, 1262.

5 Himmelfarb, E. H., Rabinowitz, J. G., Kinkhabwala, M. N., and Becker, J. A., Fournal of Urology, 1972, 108, 846.

\section{Nasal Polyps}

The respiratory mucous membrane lines nearly the whole of the nasal cavities and all the nasal accessory sinuses. Its function as an air conditioner almost constitutes it an organ in its own right, and to fulfil this function it produces an enormous amount of viscid secretion. This secretion is moved by ciliary action from the interior of the sinuses into the nasal cavities and thence to the nasopharynx, where it accumulates until an act of swallowing is evoked. The mucus is then passed into the alimentary canal and not, as is commonly believed, into the respiratory tract.

The particles of atmospheric organic and inorganic matter which adhere to the mucus in passing through the nose are digested and destroyed in the stomach and intestines. The mucus also helps to adjust the temperature and humidity of the inhaled air. The constant production of mucus is mediated by a blood-supply capable of rapid and considerable variation. In the corium of the mucosa is a vast and largely dormant network of capillaries, arterioles, and venules finely adjusted to dilate or contract in a natural response to atmospheric conditions, but also to other stimuli, psychogenic, infective, and allergic. The respiratory mucosa is perhaps the commonest site of hypersensitivity reactions, ranging from the almost specific disease of hay fever to the even more common miseries of perennial bouts of sneezing and watery rhinorrhoea.

The common factor is an exudative process as the walls of the numerous peripheral blood vessels become permeable and fluid passes from the blood stream into the tissues. Sometimes a generalized oedema occurs, resulting in a wet, pale, and thick mucosa pouring fluid from its surface in a watery flow. At other times, for unknown reasons, the fluid 
localizes in a small area. Equally obscurely, this usually occurs in the mucosa of the ethmoidal region, and then, it is thought by gravity and the suction effect of inspiration, these oedematous areas are drawn downward into the nasal cavity as polypoidal excrescences, pedunculated or sessile. Occasionally, the process occurs inside the maxillary sinus, and the oedematous mass may make its way out through the ostium into the nasal cavity and ultimately through the posterior choana into the nasopharynx, a type known as an antrochoanal polypus. Nasal polyposis may be associated with pyogenic infection, but in view of the relative frequency of both conditions the combination is unusual. It is unfortunately rarely possible to identify a specific allergen for either form of allergic reaction, and in a known case with an allergic diathesis it is impossible to forecast which, if either, type of reaction will occur.

The dominant symptom of nasal polyposis is increasing nasal obstruction, often bilateral. The patients suffering from nasal polypi rarely also suffer from sneezing attacks and rhinorrhoea, in spite of their commonly believed similar allergic aetiology.

Treatment with antihistamines would seem reasonable, but is unfortunately of little value in established cases. It is doubtful whether steroid therapy is generally justifiable, but it has been tried. From time to time beneficial results are reported. ${ }^{1}$ However, none of the suggested procedures has received much support. Effectively, removal of the obstructing masses is still the method of choice, though techniques vary.

In many cases there is only one pedunculated polypus hanging from an ethmoidal cell. Careful removal of this under a local anaesthetic using a snare is a relatively simple procedure and may never be followed by recurrence. When several pedunculated polypi are present, the same technique can be used. With a clean cutting pull-through snare the trauma is minimal. Some surgeons prefer an avulsing snare, ${ }^{2}$ but this implies an uncontrolled tearing technique which seems unnecessary in these cases. Antrochoanal polypi can also be removed by snare or fonceps (though a general anaesthetic is sometimes needed) and frequently never recur.

When polypi do recur, and especially when they are numerous and sessile, more radical measures must be used. An intranasal ethmoidectomy, attempting to remove all the mucosa at risk, is an excellent operation. ${ }^{2}$ It is not an easy one, especially if septal or other anatomical abnormalities are present, but a careful and painstaking technique taking plenty of time in visualizing and identifying structures will yield gratifying results.

External approaches to the ethmoidal region have been described and advocated. ${ }^{3}$ If the frontal sinus is involved, an external approach is essential. ${ }^{4}$ Good access to the area of disease is obtained through the classical incision in the inner canthus region or the Patterson incision below the eye. Those who advise and use an external approach are satisfied that they get a better result than with an intranasal technique, but a carefully performed operation by either method should secure a good result. Unfortunately, it is virtually impossible to remove every square centimetre of ethmoidal mucosa by any surgical operation and guarantee complete freedom from a recurrent polypus. In some cases polypi re-form almost like malignant tumours, but happily such cases are rare. The disease is not malignant and not in itself mortal, but it causes a good deal of misery. In any case of continuing and increasing nasal obstruction, nasal polyposis is a likely diagnosis, and in the early stages it can readily be treated.

1 Taylor, L. R. S., fournal of Laryngology, 1973, 87, 103.

2 Ballantyne, J., fournal of Laryngology, 1973, 87, 107.

3 Hughes, R. G., Fournal of Laryngology, 1973, 87, 11.

4 Ellis, M., fournal of Laryngology, 1954, 63, 478.

\section{Monkeys for Research}

In recent years the number of monkeys used for biomedical purposes has greatly increased, mainly in the production and testing of vaccines. Most monkeys used in laboratories are captured from their natural habitats. It has been estimated, for example, that the number imported into the United States during 1966 was greater than $100,000^{1}$ and into the United Kingdom during 1968 over 11,000. ${ }^{2}$

Many of the imported monkeys are in poor condition. They are frequently infected with pathogenic microorganisms, some of them transmissible to man, occasionally with fatal consequences. It is essential, therefore, to quarantine and condition the animals after they arrive at their destination and before they are used for experimental work or the production of vaccine. . $^{3-5}$ During a conditioning period of 12 weeks it is not unusual to lose at least $20 \%$ of each intake mainly from bacterial, viral, and parasitic infections and probably "stress" resulting from capture and transportation. The principal bacterial infections are those caused by salmonellae, shigellae and Mycobacterium tuberculosis, but the most serious microbial hazards to personnel are those created by viruses. Among these are rabies, ${ }^{67}$ Herpesvirus simiae (" $B$ " virus), 8 and the Marburg agent of vervet monkeys.9 To prevent the spread of rabies to Britain the importation of all mammals is now strictly controlled, ${ }^{10}$ and animals from abroad may be held only in quarantine premises authorized by the Minister of Agriculture, Fisheries, and Food.

Though the recommended precautions ${ }^{411}$ designed to reduce the risks associated with the handling of wild-caught monkeys or their tissues no doubt confer a high degree of safety, these risks could be further reduced by using animals bred from healthy stock under controlled conditions, just as other laboratory animals and farm animals are produced. Furthermore, the use of healthy laboratory-bred animals would reduce the numbers required for scientific purposes simply by decreasing the wastage of tissues and the frustration of experiments owing to infective agents.

Research in developmental biology, including fetal development and infant growth, will continue to be an important field of study, and many species of animals, including monkeys, will be needed for the study of problems that for ethical reasons cannot be undertaken in man. ${ }^{12}$ The demand by research workers for pregnant and newborn monkeys has lately increased, and initially attempts were made to satisfy it by importing wild-caught pregnant animals. But it was soon found that this method of obtaining them caused high wastage from abortion and stillbirths. Breeding under controlled conditions largely overcomes these problems. Moreover, when accurately timed pregnancies are required it is essential to have access to colony-bred animals with a known reproductive history.

An important aspect of using animals for experiments which the research worker must not ignore, and which is 